\title{
Lactobacillus paracasei 28.4 reduces in vitro hyphae formation of Candida albicans and prevents the filamentation in an experimental model of Caenorhabditis elegans
}

\author{
Patrícia Pimentel de Barros ${ }^{\mathrm{a}}$, Liliana Scorzoni ${ }^{\mathrm{a}}$, Felipe de Camargo Ribeiro ${ }^{\mathrm{a}}$, \\ Luciana Ruano de Oliveira Fugisaki ${ }^{\mathrm{a}}$, Beth Burgwyn Fuchs ${ }^{\mathrm{b}}$, Eleftherios Mylonakis ${ }^{\mathrm{b}}$, \\ Antonio Olavo Cardoso Jorge ${ }^{a}$, Juliana Campos Junqueira ${ }^{a}$, Rodnei Dennis Rossoni ${ }^{a}$,*

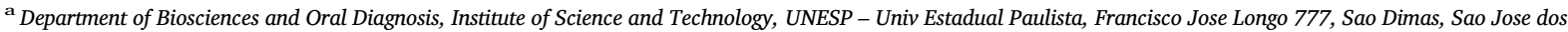 \\ Campos, CEP: 12245-000, SP, Brazil \\ ${ }^{\mathbf{b}}$ Division of Infectious Diseases, Rhode Island Hospital, Alpert Medical School of Brown University, Providence, RI, 02903, USA
}

\section{A R T I C L E I N F O}

\section{Keywords:}

Probiotics

Lactobacillus

Gene expression

Pathogenesis

Yeasts

Candida albicans

\begin{abstract}
A B S T R A C T
The objective of this study was to evaluate the influence of microbe-microbe interactions to identify a strain of Lactobacillus that could reduce the filamentation of Candida albicans ATCC 18804 using in vitro and in vivo models. Thus presenting a probiotic effect against the fungal pathogen. First, we analyzed the ability of 25 clinical isolates of Lactobacillus to reduce filamentation in C. albicans in vitro. We found that L. paracasei isolate 28.4 exhibited the greatest reduction of $C$. albicans hyphae $(p=0.0109)$. This reduction was confirmed by scanning electron microscopy analysis. The influence of $C$. albicans filamentation was found to be contributed through reduced gene expression of filament associated genes (TEC1 and UME6). In an in vivo study, prophylactic provisions with L. paracasei increased the survival of Caenorhabditis elegans worms infected with C. albicans ( $p=0.0001$ ) by $29 \%$. Prolonged survival was accompanied by the prevention of cuticle rupture of $27 \%$ of the worms by filamentation of $C$. albicans, a phenotype that is characteristic of $C$. albicans killing of nematodes, compared to the control group. Lactobacillus paracasei isolate 28.4 reduced the filamentation of $C$. albicans in vitro by negatively regulating the TEC1 and UME6 genes that are essential for the production of hyphae. Prophylactic provision of Lactobacillus paracasei 28.4 protected C. elegans against candidiasis in vivo. L. paracasei 28.4 has the potential to be employed as an alternative method to control candidiasis.
\end{abstract}

\section{Introduction}

Approximately 1.4 million people have suffered from fungal infections worldwide during the last decade [1]. Some of these mycoses are difficult to diagnose and treat and may cause sequelae [2]. Candida species are highlighted because of their high incidence of opportunistic fungal infections, Candida albicans being the main causative agent of these infections $[3,4]$. The polymorphic fungi $C$. albicans belongs to the human microbiome and, in normal conditions, does not affect the host $[5,6]$. However, under some circumstances, such as incidence of host immunosuppression, these fungi can cause infections ranging from superficial (oral and vaginal candidiasis) to systemic infection [5-7].

Various mechanisms contribute to the virulence of $C$. albicans including adhesion/invasion molecules, secreted enzymes such as hydrolases, biofilm formation, and yeast-to-hyphae transition $[6,8,9]$. The dimorphism of $C$. albicans is the ability of this fungus to transition from yeast to hyphae morphology, and both forms are important for host infection: the yeast is considered to be important for the dissemination of the infection, while the hyphae have an essential role during tissue invasion $[6,10,11]$. The morphological transition is influenced by some factors such as $\mathrm{pH}$, temperature $\left(37^{\circ} \mathrm{C}\right)$, nutrients, and serum [12]. In addition, the morphological transition relies on a complex pathway with multiple steps and is synchronized by transcription factors such as the genes UME6 and TEC1 that correspond to positive regulators of the process [13-15].

The limited arsenal of antifungal drugs and their toxicity, together with the antifungal resistance mechanisms developed by this microorganism, led to the necessity of developing efficient strategies for

\footnotetext{
* Corresponding author.

E-mail addresses: barrosdnapp@yahoo.com.br (P.P. de Barros), liliscorzoni@yahoo.com.br (L. Scorzoni), felipe_c_ribeiro@hotmail.com (F.d.C. Ribeiro), luciana.fugisaki@ict.unesp.br (L.R.d.O. Fugisaki), helen_fuchs@brown.edu (B.B.Fuchs), emylonakis@lifespan.org (E. Mylonakis), olavojorge@fosjc.unesp.br (A.O.C. Jorge), juliana@fosjc.unesp.br (J.C. Junqueira), rodnei.rossoni@fosjc.unesp.br (R.D. Rossoni).
} 
antifungal therapy [16]. One of these strategies is to target the fungal virulence mechanisms using anti-virulence agents $[12,17]$. The development of anti-virulence agents would bring new and a high number of potential targets, preserve the host normal flora, and potentially decrease the antifungal resistance index [12,17].

Recent reports demonstrate the importance of the filamentation process of $C$. albicans for the development of anti-virulence agents [12]. The search for C. albicans anti-filamentation substances can be found in different sources such as chemical libraries of small molecules [18], natural compounds obtained from plants [19], peptides isolated from bacteria [20], and through microorganism-microorganism interactions $[21,22]$. We focus on the latter.

Lactobacillus is a Gram-positive bacterium known to interact with other microbes through probiotic effects. The Lactobacillus spp.-C. albicans interaction is described in different studies [23-25]. The possible mechanisms that Lactobacillus spp. could use to prevent $C$. albicans colonization include the secretion of substances that decrease fungal adhesion (biosurfactants), the production of bacteriocin-like compounds to suppress fungal proliferation, host immunomodulation, and the production of hydrogen peroxide and lactic acid that interfere with hyphal formation [22,23,26,27]. Based on this, the use of probiotics would be an interesting strategy to develop anti-filamentation agents.

In vivo studies can be valuable in providing a better understanding of the mechanisms involved during these microorganism-microorganism interactions. Caenorhabditis elegans is described as an infection model for Candida spp. in the study of new antifungal compounds, anti-virulence agents, and immunomodulatory activity [28-32]. Additionally, C. elegans permits the study of $C$. albicans filamentation because hyphae formation can be observed after the ingestion of this yeast; the hyphae grow through the worm cuticle causing $C$. elegans death [33,34].

In this context, the aim of this study was to evaluate the effect of Lactobacillus isolates on C. albicans in relation to the filamentation process, analyzing in vitro and in vivo approaches. In addition, the regulation of this mechanism was analyzed, evaluating genes that are important for the C. albicans filamentation process.

\section{Material and methods}

\subsection{Strains and growth conditions}

In this study, we used the reference strain C. albicans ATCC 18804 and 25 clinical strains of Lactobacillus: 20 strains of L. paracasei, 4 strains of L. rhamnosus and 1 strain of $L$. fermentum (Table 1 ). The Lactobacillus strains were isolated from the human oral cavity of an individual without caries, per the approval of the Ethics Committee under protocol 560.479 and identified in a previous study of our group [35]. C. albicans was cultured for $18 \mathrm{~h}$ at $37^{\circ} \mathrm{C}$ in yeast nitrogen base broth (YNB; Difco, Detroit, MI, USA) supplemented with $100 \mathrm{mM}$ of glucose. The Lactobacillus strains were cultured in Lactobacillus ManRogosa-Sharpe broth (MRS broth; Difco, Detroit, USA) for $24 \mathrm{~h}$ at $37^{\circ} \mathrm{C}$ under microaerophilic conditions. The suspension densities were determined with a spectrophotometer (B582, Micronal, Sao Paulo, Brazil) and were then diluted to concentrations of $10^{7}$ cells $/ \mathrm{mL}$. The number of cells in the inoculum was confirmed by counting the $\mathrm{CFU} / \mathrm{mL}$ following plating on Sabouraud dextrose agar (SDA; Himedia, Mumbai, India) for C. albicans and MRS agar (Difco, Detroit, MI, USA) for Lactobacillus.

\subsection{Selection of the best Lactobacillus strain to reduce filamentation: In vitro study}

\subsubsection{Induction of filamentation by $C$. albicans}

To select the Lactobacillus strain with the prominent anti-Candida activity, a filamentation assay was used to compare the effects posed by the 25 isolates from the oral cavity. This assay was performed in 24well microtiter plates (TPP ${ }^{\varpi}$, Trasadingen, Switzerland) following a
Table 1

Clinical strains of Lactobacillus used in this study.

\begin{tabular}{ll}
\hline Species & Strain designation \\
\hline L. paracasei & 1.1 \\
L. paracasei & 3.1 \\
L. paracasei & 4.2 \\
L. paracasei & 6.2 \\
L. paracasei & 7.5 \\
L. paracasei & 8.4 \\
L. paracasei & 11.6 \\
L. rhamnosus & 13.1 \\
L. paracasei & 15.8 \\
L. paracasei & 16.4 \\
L. paracasei & 17.1 \\
L. rhamnosus & 19.3 \\
L. rhamnosus & 19.9 \\
L. paracasei & 21.4 \\
L. paracasei & 23.4 \\
L. paracasei & 24.1 \\
L. paracasei & 25.4 \\
L. paracasei & 26.1 \\
L. paracasei & 27.1 \\
L. paracasei & 28.4 \\
L. paracasei & 30.1 \\
L. fermentum & 31.4 \\
L. rhamnosus & 36.4 \\
L. paracasei & 37.1 \\
L. paracasei & 39.2 \\
\hline
\end{tabular}

methodology described previously [36,37] with some modifications. The following groups were evaluated: $C$. albicans + Phosphate-buffered saline (PBS/control group) and C. albicans + Lactobacillus spp. The PBS buffer was diluted to a $1 \mathrm{X}$ working concentration that contains $137 \mathrm{mM}$ $\mathrm{NaCl}, 2.7 \mathrm{mM} \mathrm{KCl}, 8 \mathrm{mM} \mathrm{Na}_{2} \mathrm{HPO}_{4}$, and $2 \mathrm{mM} \mathrm{KH}_{2} \mathrm{PO}_{4}$. The experiment was performed as three biological replicates independently in triplicate.

In a 24-well culture plate, $1 \mathrm{~mL}$ of distilled water was mixed with $10 \%$ fetal bovine serum, $50 \mu \mathrm{L}$ of BHI broth (Difco, Detroit, MI, USA) and $100 \mu \mathrm{L}$ of standardized C. albicans suspension. Fifty microliters of standardized Lactobacillus spp. suspension was also added to the experimental group, while $50 \mu \mathrm{L}$ of PBS was added to the control group. The plates were incubated at $37^{\circ} \mathrm{C}$ under a partial pressure of $5 \% \mathrm{CO}_{2}$. After $24 \mathrm{~h}$ of incubation, $50 \mu \mathrm{L}$ of the inoculum was transferred to glass slides with 10 previously demarcated fields on the back of the slide and observed under a light microscope at 40X magnification. The images were analyzed for the $C$. albicans morphology, and 10 microscopic fields per slide were chosen to quantify the hyphae. The following scores were used to delineate the number of hyphae present in each microscopic field: 0 (no hyphae), 1 (1-15 hyphae), 2 (16-30 hyphae) and 3 (more than 30 hyphae).

\subsubsection{Measurement of $\mathrm{pH}$ values}

The $\mathrm{pH}$ of the media during the filamentation process were tested under the same conditions as the filamentation assay described above. After $24 \mathrm{~h}$ of incubation in the 24-well culture plates, the supernatant from each well was collected, and the $\mathrm{pH}$ value was measured using a pH meter (Mettler, Toledo, Ohio, USA). Four wells were measured per group, and the experiment was done at three different times.

\subsubsection{Analysis of filamentation assay by scanning electron microscopy (SEM)}

The L. paracasei 28.4 strain demonstrated the greatest ability to reduce hyphae production, and all the experiments of this study were conducted with this strain. Acrylic resin discs measuring $8 \mathrm{~mm}$ in diameter were placed on a 24-well plate for filamentation assay as previously described. After $24 \mathrm{~h}$, the specimens were fixed in $1 \mathrm{~mL}$ of $2.5 \%$ glutaraldehyde for $1 \mathrm{~h}$. The specimens were then dehydrated in an increasing ethanol series $(10,25,50,75$ and 90\%) for 20 min each, followed by immersion in $100 \%$ alcohol for $1 \mathrm{~h}$. The plates were kept in an 
oven at $37^{\circ} \mathrm{C}$ for $24 \mathrm{~h}$ to permit complete drying of the specimens. After drying, the specimens were transferred to aluminum stubs and sputtered with gold for $160 \mathrm{~s}$ at $40 \mathrm{~mA}$ (Denton Vacuum Desk II). The specimens were examined and photographed using a JEOL JSM5600 scanning electron microscope at the Institute of Science and Technology, UNESP (Univ Estadual Paulista). These experiments were performed at two different times with three assays per group.

\subsubsection{Quantitative real-time polymerase chain reaction ( $q P C R$ )}

The qReal-time PCR assay was performed in the same conditions described in the in vitro filamentation assay. Total RNA was isolated with the TRIzol reagent (Ambion, Inc., Carlsbad, CA, USA). The extracted total RNA (700 ng) was treated with DNase I (Turbo DNase Treatment and Removal Reagents - Ambion Inc., Carlsbad, CA, USA) and transcribed into complementary DNA (cDNA) using SuperScript ${ }^{\circ}$ IV First-Strand Synthesis SuperMix for qRT-PCR Kit (Invitrogen $^{\mathrm{TM}}$, Carlsbad, CA, USA), according to the manufacturer's instructions.

Transcribed cDNAs were amplified for the relative quantification of TEC1 and UME6 gene expression levels in relation to the concentration of the reference gene (ACT1). In our study, three reference genes, ACT1, $P M A 1$ and $R P P 2 B$, were tested in all the experimental groups. The results were analyzed at http://www.leonxie.com/referencegene.phpe, and the reference gene chosen was ACT1. All the primers used in the present study were described previously $[38,39]$.

Quantitative real-time PCR was conducted using a Platinum ${ }^{\circledR}$ SYBR ${ }^{\oplus}$ Green qPCR SuperMix-UDG Kit (Applied Biosystems, Framingham, MA, USA) in the StepOnePlus ${ }^{\mathrm{TM}}$ apparatus (Applied Biosystems, Framingham, MA, USA). The $2^{-} \Delta \Delta^{\mathrm{CT}}$ method was used to analyze the relative changes in gene expression from the quantitative RT-qPCR experiment [40].

\subsection{Lactobacillus paracasei 28.4 interferes in filamentation of C. albicans: in vivo study}

\subsubsection{C. elegans strain}

C. elegans glp-4, sek-1 strain was used in this study with the methodologies described by Oh et al. [41], Kim et al. [42] and Peleg et al. [43]. Mixed infections with Lactobacillus and C. albicans were induced in C. elegans in all experiments (survival and filamentation assays). As control groups, a monotypic infection was induced in $C$. elegans by inoculation only of C. albicans, Lactobacillus, or E. coli OP50.

\subsubsection{Preparation of conditioning plates with probiotic bacteria}

To prepare probiotic-conditioning plates, L. paracasei 28.4 was grown in Man-Rogosa-Sharpe (MRS) medium (Difco, Detroit, MI, USA) at $37^{\circ} \mathrm{C}$ for $24 \mathrm{~h}$. Following five washes with M9 medium, $500 \mu \mathrm{L}$ of $L$. paracasei 28.4 suspension $\left(1.0 \times 10^{9} \mathrm{CFU} / \mathrm{mL}\right)$ was spread on a Nematode Growth Medium (NGM) plate and dried for $3 \mathrm{~h}$ at room temperature. Conditioning plates were stored at $4{ }^{\circ} \mathrm{C}$ and used within 1 week. E. coli strain OP50 was used as the control, and the same protocols were followed. The M9 medium consists of $3 \mathrm{~g} \mathrm{KH}_{2} \mathrm{PO}_{4}, 6 \mathrm{~g}$ $\mathrm{Na}_{2} \mathrm{HPO}_{4}, 0.5 \mathrm{~g} \mathrm{NaCl}, 1 \mathrm{~g} \mathrm{NH} \mathrm{Cl}_{4}$ and $0.25 \mathrm{~g} \mathrm{MgSO}_{4} \cdot 7 \mathrm{H}_{2} \mathrm{O}$ in $1000 \mathrm{~mL}$ of water. The NGM plate consists of $2.5 \mathrm{~g}$ peptone, $3 \mathrm{~g} \mathrm{NaCl}, 20 \mathrm{~g}$ agar, $1 \mathrm{mM} \mathrm{CaCl} 2,1 \mathrm{mM} \mathrm{MgSO}_{4}, 25 \mathrm{mM} \mathrm{KPO}, 5 \mu \mathrm{g} / \mathrm{mL}$ cholesterol in $1000 \mathrm{~mL}$ of water.

\subsubsection{Effects of Lactobacillus on experimental candidiasis in C. elegans: survival assay}

First, C. elegans nematodes were cultured on NGM plates with a $12 \mathrm{~h}$ culture of E. coli OP50 $\left(10^{8} \mathrm{CFU} / \mathrm{mL}\right)$ for $48 \mathrm{~h}$ at $25^{\circ} \mathrm{C}$. After this time, the nematodes were washed twice with M9 buffer using centrifugation.

Liquid killing assay and the different times of infection were performed using published methods with some modifications [43]. A synchronous population of worms is necessary in order to eliminate variation in results due to age differences. Thereby, the worms were synchronized in young adult stage. Young adult worms were placed on conditioning plates with $L$. paracasei 28.4 at $25^{\circ} \mathrm{C}$ for $2 \mathrm{~h}$. After this period, the nematodes were washed with M9 buffer and placed in brain heart infusion (BHI - Difco, Detroit, MI, USA) plates containing C. albicans for $2 \mathrm{~h}$. Infected worms were then washed twice with M9 medium and transferred into wells of a 6-well microtiter dish (50 worms per well). Each well contained $2 \mathrm{~mL}$ of liquid assay medium (20\% BHI and $80 \%$ M9). For the control groups (E. coli + E. coli, E. coli + C. albicans and $L$. paracasei $+E$. coli), the worms were infected with two subsequent infections of $2 \mathrm{~h}$ each as in the L. paracasei $+C$. albicans group described above. The plates were sealed with a breathe easy membrane (Sigma Aldrich, St. Louis, MO) and incubated at $25^{\circ} \mathrm{C}$ for 10 days. Nematodes were observed daily under a stereomicroscope and were considered dead when they did not move after being touched with a platinum loop. This assay was performed as three independent experiments with $n=6$ wells per group.

\subsubsection{Study of C. albicans filamentation in C. elegans treated with Lactobacillus}

The filamentation assay was performed according Peleg et al. [43]. The $C$. elegans nematodes were provided with $L$. paracasei 28.4 as a food source for $4 \mathrm{~h}$, and then $C$. albicans was used to infect the worms. For this, the nematodes were fed $C$. albicans that were seeded on the surface of BHI agar (Difco, Detroit, MI, USA) and incubated at $25^{\circ} \mathrm{C}$ for $4 \mathrm{~h}$. The nematodes were then washed with M9 buffer and transferred by pipette into each well of a 6-well culture of cells containing $2 \mathrm{~mL}$ of liquid medium (80\% M9 and 20\% BHI) plates. The plates were incubated at $25^{\circ} \mathrm{C}$ and analyzed after $60 \mathrm{~h}$ to identify nematodes with C. albicans filamentation. This analysis was conducted using a stereomicroscope (Nikon SMZ645, Minato, Tokyo, Japan) to observe a rupture in the cuticle of the nematode by the filamentation of $C$. albicans. The differences in filamentation were compared between groups of nematodes infected with $L$. paracasei 28.4 and C. albicans and infected only with $C$. albicans. This assay was performed in triplicate.

\subsection{Statistical analysis}

A statistical analysis was performed using GraphPad Prism 6 software (GraphPad Software, Inc., La Jolla, CA, USA). The scores obtained through in vitro filamentation analysis were compared using the MannWhitney test. The percent survival of $C$. elegans was plotted, and statistical analysis was performed using the Kaplan-Meier test. Student's $t$ test was used to compare the filamentation assay with C. elegans. A $P$ value $\leq 0.05$ was considered significant.

\section{Results}

First, we carried out an in vitro assay as a screening with 25 clinical isolates of Lactobacillus spp. to select the strain with the greatest capacity to interfere with the formation of $C$. albicans hyphae. Among the 25 strains analyzed, only 8 could significantly reduce the formation of C. albicans hyphae, as shown in Fig. 1.

We observed many $C$. albicans hyphae in the control group with PBS. However, we verified that hyphal formation was significantly reduced when $C$. albicans was incubated with Lactobacillus strains 13.1 ( $p=0.0209), 19.3$ ( $p=0.0420), 26.1(p=0.0420), 25.4(p=0.0290)$, $30.1(p=0.0209), 27.1(p=0.0290), 19.9(p=0.0290)$ and 28.4 ( $p=0.0109$ ) compared to the control group. Because L. paracasei strain 28.4 resulted in lower $C$. albicans filamentation scores compared to the other strains, it was chosen for subsequent assays.

To invalidate the hypothesis that the $C$. albicans hyphae were inhibited by the reduction in the $\mathrm{pH}$ of the medium due to the action of Lactobacillus, which are lactic acid bacteria, we compared the $\mathrm{pH}$ of the control group and the group associated with strain 28.4. Although the $\mathrm{pH}$ value decreased in the interaction group with $L$. paracasei 28.4 , it was not significant $(p=0.0643)$, suggesting that there are metabolites secreted by the probiotic strain capable of interfering with the 


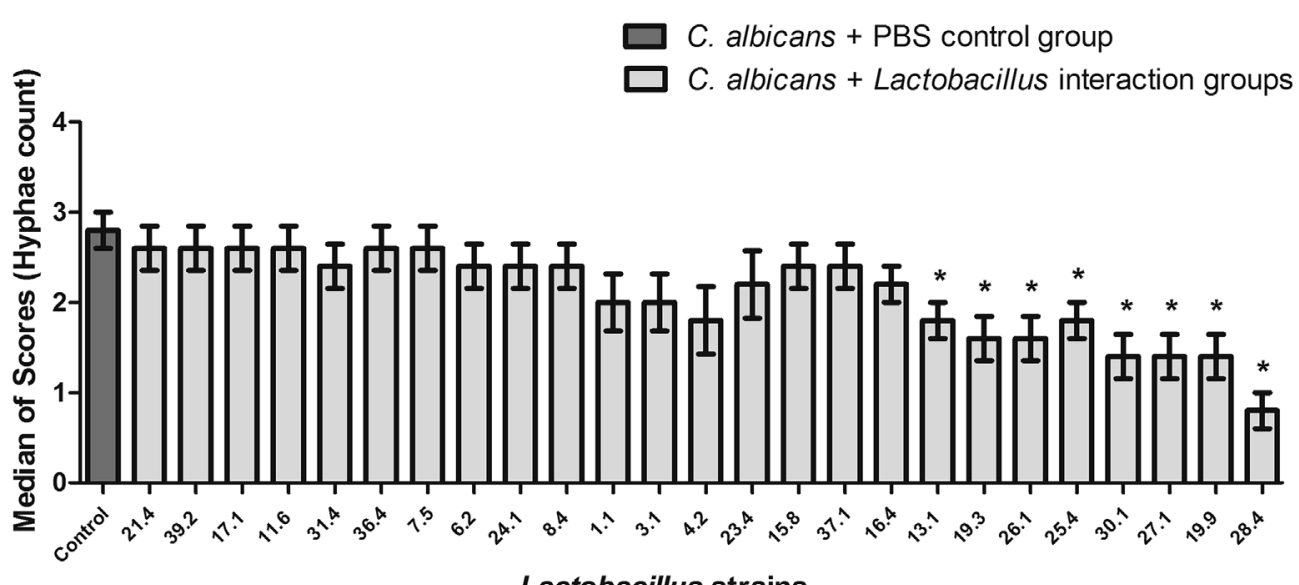

\section{Lactobacillus strains}

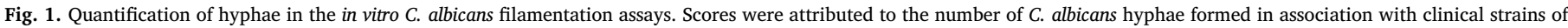
Lactobacillus or with PBS (control group). Mann-Whitney test, $p \leq 0.05$.

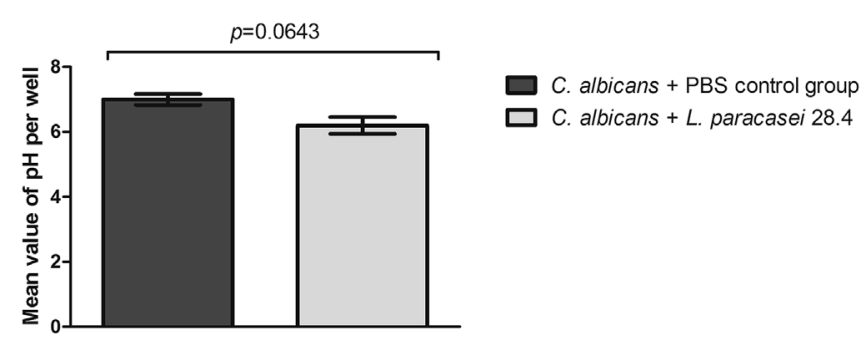

Fig. 2. The reduction of hyphae was not caused by $\mathrm{pH}$ change in the medium. Mean and standard deviations for the $\mathrm{pH}$ value per well in the filamentation assay. Student's $t$-test, $p \leq 0.05$.

formation of hyphae (Fig. 2). In addition, the SEM analysis revealed a large number of hyphae in the control group of $C$. albicans, as opposed to large numbers of yeast in the group associated with L. paracasei 28.4, confirming the results obtained in the previous assay (Fig. 3).

To elucidate the mechanisms involved in the action of $L$. paracase $i$ 28.4 on the filamentation of $C$. albicans, we did quantification of transcriptional genes such as TEC1 and UME6 by qPCR. These genes were downregulated with 2.5- and 3.0-fold decreases, respectively, when compared to the control (Fig. 4). Both TEC1 and UME6 are required for the morphogenesis of $C$. albicans and play key roles in this important virulence factor.

In the in vivo assays, we first examined whether $L$. paracasei strain 28.4 could influence the life span of $C$. elegans. As a control, we employed the Gram-negative bacterium E. coli strain OP50 that is nonpathogenic to $C$. elegans. We found no significant difference in the viability of $C$. elegans exposed to E. coli OP50 or 28.4 strain in the liquid assays (Fig. 5A) ( $p=0.1270$ for $L$. paracasei $+E$. coli group compared with worms fed only OP50). Therefore, we concluded that 28.4 strain does not significantly reduce the lifespan of $C$. elegans.

We hypothesized that despite the ability of $C$. albicans to kill $C$. elegans individually, a combined infection with $L$. paracasei might lead to attenuated killing compared with $C$. albicans alone due to the fact that hyphae formation by $C$. albicans inhibition of $C$. elegans contributes to the death of the worms when filaments puncture the cuticle. We observed that when C. elegans was sequentially exposed for $2 \mathrm{~h}$ with $L$. paracasei, followed by $C$. albicans on separate agar plates, and then transferred into standard liquid medium, the death of the nematodes was significantly attenuated ( $29 \%$ increase in survival) compared with that observed with $C$. albicans infection alone ( $p=0.0001$ ) (Fig. 5A).

Given the importance of filamentation in the pathogenesis of $C$. albicans infection in mammals and $C$. elegans, we sought to assess the consequences of prior exposure to $L$. paracasei on the filamentation of $C$. albicans within the worms. When $C$. elegans was infected with C. albicans and exposed to a liquid environment, the majority of the nematodes died due to the penetration of $C$. albicans filaments through the nematode cuticles (Fig. 5B). C. albicans filamentation within C. elegans begins within $24 \mathrm{~h}$ of liquid-medium exposure and peaks by $60 \mathrm{~h}$. Remarkably, when nematodes were exposed to $L$. paracasei prior to C. albicans infection, there was a reduction in the number of worms exhibiting filaments (27\%), but it was not statistically significant $(p=0.1012)$ (Fig. 5B; Fig. 6).

\section{Discussion}

A variety of commercially available probiotic preparations are
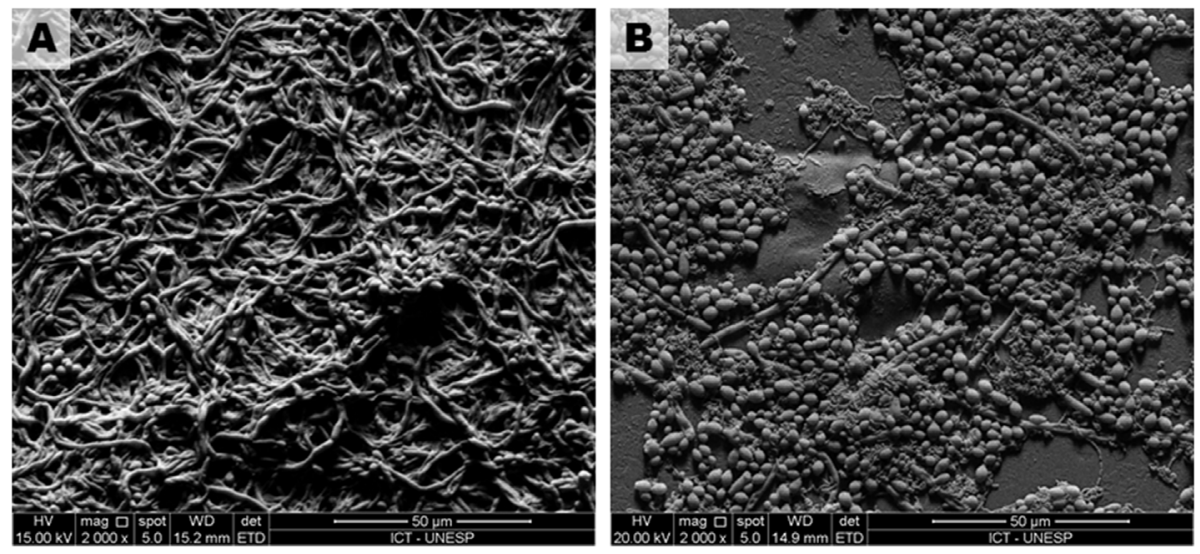

Fig. 3. SEM of filaments formed in vitro. A. Control group of C. albicans + PBS: the presence of numerous hyphae is verified, B. Group interaction of C. albicans + L. paracasei 28.4 cells. In the interaction group, it is possible to observe a reduction in the number of hyphae of $C$. albicans and an intimate interaction between C. albicans and Lactobacillus cells. Magnification: 2000X. 




A

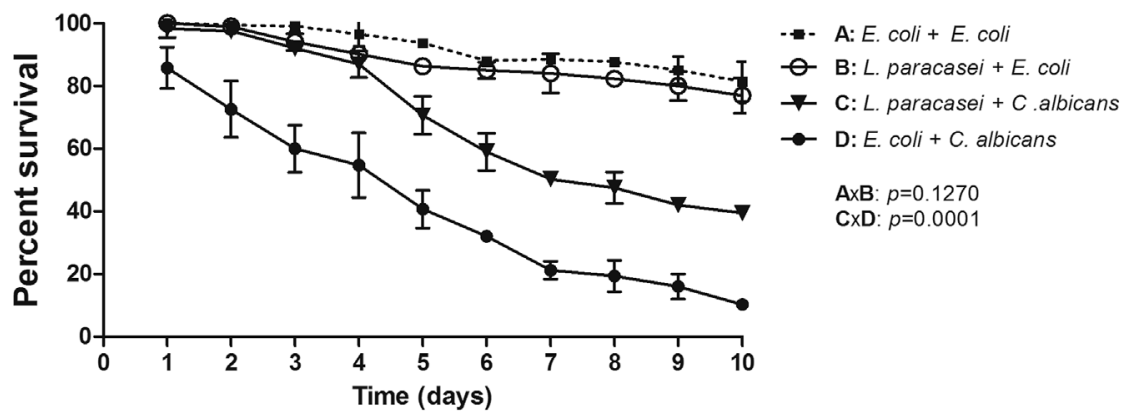

B

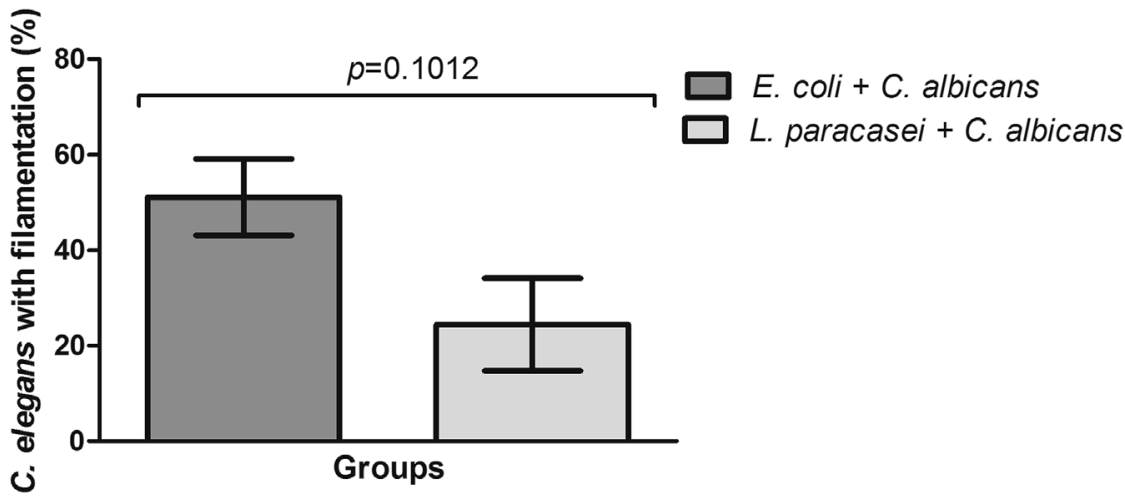

Fig. 4. Relative quantification of TEC1 and UME6 in monotypic and mixed groups of $C$. albicans associated with L. paracasei 28.4. Each gene, UME6 or TEC1, was normalized and compared with $C$. albicans + PBS (control). Values were expressed as the mean and standard deviation. Student's $t$-test was used to compare gene expression among the groups studied $(p \leq 0.05)$.
Fig. 5. The probiotic bacterium L. paracasei 28.4 prolonged the survival of $C$. elegans nematodes infected with $C$. elegans and decreased the frequency of nematodes observed with filaments protruding through the cuticle. A. Liquid killing assays of C. elegans ( $\mathrm{n}=50$ per well) $g l p-4$ worms infected with C. albicans ATCC 18804 after conditioning with $L$. paracasei 28.4. Survival statistic: $p=0.0001$ for $L$. paracasei + C. albicans group compared to worms that were only fed C. albicans (E. coli $+C$. albicans group). $P$ values were obtained from the repetition of the most representative experiment. Kaplan-Meier test, $p \leq 0.05$. B. Mean and standard deviation of worms with $C$. albicans filaments in the liquid medium assay. Student's $t$-test was used to compare the worms with $C$. albicans filaments between the groups studied ( $p \leq 0.05$ ). comprised of strains of Lactobacillus or Bifidobacterium isolated from human feces to facilitate compatibility with the human intestinal microbiota [44]. Among the bacteria that show promise to be used as probiotics in the oral cavity, Lactobacillus spp. has been the subject of much research because it is a microorganism belonging to the normal oral microbiota able to colonize and modulate this microbiota, thus inhibiting the growth of several pathogens such as Candida yeasts $[27,45]$. In this study, we screened the capacity of 25 clinical isolates of
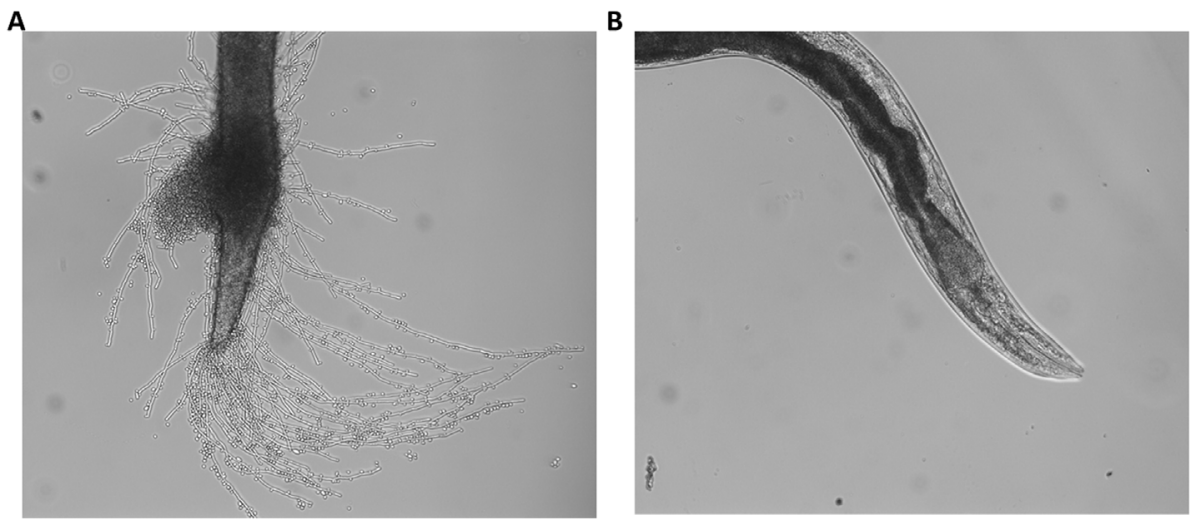

Fig. 6. Prophylactic provisions of $L$. paracasei reduce the filamentation of $C$. albicans in a model of co-infection with C. elegans. A. Control group of $C$. albicans: it is possible to observe the formation of hyphae rupturing the cuticle of the nematodes ( $60 \mathrm{~h}$ after infection). B. L. paracasei + C. albicans group: formation of few or no hyphae of $C$. albicans can be observed after $60 \mathrm{~h}$ of infection. Magnification: 100X. 
Lactobacillus to reduce filamentation in C. albicans in vitro for use as a potential probiotic in the oral cavity.

By analyzing clinical Lactobacillus isolates from the oral cavity of healthy patients, we found that some strains provide better reduction of C. albicans filamentation than others. We found that eight Lactobacillus isolates could reduce $C$. albicans hyphae formation, and $L$. paracasei 28.4 showed the greatest amount of reduction. This fact could be due to the Lactobacillus production of antimicrobial molecules such as bacteriocins, since the reduction of the $\mathrm{pH}$ was not significant $[46,47]$.

The in vitro reduction of hyphae formation by strain $L$. paracasei 28.4 that we observed suggests that this strain could be a highly promising potential probiotic that could be used to control C. albicans infections, since the hyphae of $C$. albicans are responsible for invasion into host tissues. Mailänder-Sánchez et al. (2017) performed a study using a multilayer of oral epithelial cells (reconstructed human oral epithelium, RHOE) and provided the first clear evidence for a protective effect of Lactobacillus rhamnosus GG (LGG) against infection with C. albicans. Pre-incubation of RHOEs with LGG protected the tissue from C. albicans-induced cell damage, characterized by a strong reduction in the levels of epithelial lactate dehydrogenase (LDH) released into the RHOE supernatant, which indicates lysis of the epithelial cells. Through histological analysis, the authors found a strong reduction in the signs of damage in the cell layer and hyphae growth into the epithelial cells, while in the absence of LGG, C. albicans invaded the whole epithelium and caused vacuoles as signs of damage.

Recent studies have also shown that Lactobacillus spp. can reduce the number of hyphae by C. albicans formed in vitro [36,37]. Lactobacilli can create an adverse microenvironment for pathogens by delivering localized antimicrobial compounds, thus impairing epithelial colonization and subsequent tissue invasion [48-50]. Metabolites released by Lactobacillus species such as sodium butyrate have been shown to suppress $C$. albicans filamentation, reducing fungal pathogenicity [51]. Noverr and Huffnagle [52] observed that supernatants obtained from $2 \mathrm{~h}$ probiotic bacteria inhibited germ tube formation in C. albicans, and complete inhibition of germination was obtained in $24 \mathrm{~h}$ supernatants. Other antimicrobial metabolites described in the literature may reduce the survival of the pathogen as a result of their synergistic actions, such as biosurfactants, organic acids, and $\mathrm{H}_{2} \mathrm{O}_{2}$ [53], bacteriocins [54] and peptide fermenticin HV6b [55].

We explored the specific effect caused by $L$. paracasei 28.4 by examining the expression of genes that are important for the $C$. albicans filamentation process, TEC1 and UME6. During the pathogenicity process of $C$. albicans, several transcriptional regulators are known to play critical roles. Importantly, two of these regulators, TEC1 and UME6, are themselves controlled at the transcriptional level by filament-inducing conditions [56,57]. UME6, a zinc finger DNA-binding protein, lies at the center of hyphal regulation in C. albicans as a necessary and sufficient factor for hyphae growth $[14,58,59]$ and as a key transcriptional target for the various signal transduction pathways that mediate hyphal induction stimuli [60]. TEC1 is involved in the regulation of hyphaespecific genes, and it is required for wild-type biofilm formation [57,61]. In addition, C. albicans TEC1 acts downstream of EFG1, a protein that plays a critical role in hyphae morphogenesis [10]. Our results demonstrated that $L$. paracasei 28.4 associated with $C$. albicans significantly downregulate these two genes.

Among the virulence factors of $C$. albicans, filamentation represents an attractive target, already validated at the genetic level, for the development of anti-virulence approaches against candidiasis. The ability of $C$. albicans to respond to a multitude of signals allows its adaptation to the environmental conditions presented by the host. Some signals can stimulate hyphae formation including neutral $\mathrm{pH}, 5 \% \mathrm{CO}_{2}$, body temperature $\left(37^{\circ} \mathrm{C}\right)$, serum, nutrient availability and embedded and microaerophilic conditions $[6,45]$. The filamentation process in C. albicans is tightly regulated, while simultaneously being highly dynamic, occurring under a wide range of environmental conditions [62,63]. Due to its activity against C. albicans filaments, L. paracasei strain 28.4 represents a new potential probiotic strain that can be used to control $C$. albicans infections.

The introduction of $C$. albicans in C. elegans causes lethal infection with intense proliferation of yeast in the intestinal tract and the subsequent formation of hyphae breaching the cuticle of the nematode [31]. Pukkila-Worley et al. [33] and Tan et al. [64] also demonstrated that the formation of hyphae by $C$. albicans is an important virulence factor to model infection in C. elegans, and some C. albicans mutant strains incapable of forming hyphae were less virulent to these nematodes. In this study, we also observed a significant inhibition of $C$. albicans filamentation by the influence of $L$. paracasei, agreeing with our in vitro results on the specific effect that isolate 28.4 has on filament formation.

The inhibition of various microbial pathogens by Lactobacillus spp. was demonstrated in in vivo studies using a C. elegans model [65-68]. Kim et al. [42] showed that using the probiotic Lactobacillus acidophilus NCFM to condition C. elegans prolongs the survival of nematodes exposed to resistant Enterococcus faecium. Recently, Li et al. [66] explored the role of Lactobacillus plantarum in modulating host responses to vancomycin-resistant Enterococcus faecium (VRE) using C. elegans. The authors found that $L$. plantarum also significantly protected the worms against VRE infection by increasing the transcription of host defense genes, such as cpr-1 and clec-60, reported to have protective roles against infections. Our previous work was consistent with these results and showed that the probiotic strain used in this study (L. paracasei 28.4) had immunomodulatory action in the Galleria mellonella model and protected the insects against $C$. albicans infection by up-regulating the genes encoding antifungal peptides, such as galiomicin and gallerymicin [27].

More specifically the mechanism of interaction between $L$. paracasei 28.4 and $C$. albicans in C. elegans model may be explained by two hypotheses. First, it was verified that the probiotic strain (L. paracasei 28.4) used in this study has direct action on C. albicans and its mechanisms of pathogenicity. Rossoni et al. [35] observed that $L$. paracasei 28.4 reduced the biofilm, hyphae formation and downregulated genes from different strains of $C$. albicans. Second, it is suggested that this strain has an indirect action by stimulating $C$. elegans defense genes such as FIP (fungus-induced protein), NLP (neuropeptide-like protein), CNC (caenacin) and GRSP (glycine-rich secreted protein) in C. albicans infections. According Pujol et al. [69] all these genes could be responsible to encode antimicrobial peptides (AMPs) involved in the innate response against fungi.

The different mechanisms used by probiotics to combat pathogens are still unclear. In addition, the differences in metabolites between Lactobacillus species merit further research. Future studies to identify the exact compounds produced by $L$. paracasei 28.4 and their mechanisms underlying the inhibition of $C$. albicans hyphae need to be conducted in more detail.

\section{Conclusion}

In summary, the clinical strains of Lactobacillus isolated from the oral cavity show varied activity against $C$. albicans filamentation. $L$. paracasei 28.4 reduced the filamentation of $C$. albicans in vitro by negatively regulating the TEC1 and UME6 genes, which are essential for the production of hyphae. $L$. paracasei 28.4 protected $C$. elegans against experimental candidiasis in vivo. L. paracasei 28.4 demonstrated the best probiotic profile compared with the other lactobacilli species tested, suggesting that it may be useful as an adjunctive therapeutic mode against oral Candida infections.

\section{Conflicts of interest}

The authors declare that they have no conflicts of interest. 


\section{Acknowledgments}

This study was supported by the Fundação de Amparo à Pesquisa do Estado de São Paulo (FAPESP) under grant numbers 2014/12458-4, 2013/25181-8 and 2015/09770-9 and by the Brown/Brazil Initiative.

\section{References}

[1] G.D. Brown, D.W. Denning, N.A. Gow, S.M. Levitz, M.G. Netea, T.C. White, Hidden killers: human fungal infections, Sci. Transl. Med. 4 (2012) 165rv13.

[2] J. Jampilek, How can we bolster the antifungal drug discovery pipeline? Future Med. Chem. 8 (2016) 1393-1397.

[3] M.A. Pfaller, D.J. Diekema, Epidemiology of invasive candidiasis: a persistent public health problem, Clin. Microbiol. Rev. 20 (2007) 133-163.

[4] J.C. Sardi, L. Scorzoni, T. Bernardi, A.M. Fusco-Almeida, Mendes Giannini MJ. Candida species: current epidemiology, pathogenicity, biofilm formation, natural antifungal products and new therapeutic options, J. Med. Microbiol. 62 (2013) 10-24.

[5] M.C. Arendrup, Epidemiology of invasive candidiasis, Curr. Opin. Crit. Care 16 (2010) 445-452.

[6] F.L. Mayer, D. Wilson, B. Hube, Candida albicans pathogenicity mechanisms, Virulence 4 (2013) 119-128.

[7] B.J. Kullberg, M.C. Arendrup, Invasive candidiasis, N. Engl. J. Med. 373 (2015) 1445-1456.

[8] G. Ramage, E. Mowat, B. Jones, C. Williams, J. Lopez-Ribot, Our current understanding of fungal biofilms, Crit. Rev. Microbiol. 35 (2009) 340-355.

[9] B. Wächtler, D. Wilson, K. Haedicke, F. Dalle, B. Hube, From attachment to damage: defined genes of Candida albicans mediate adhesion, invasion and damage during interaction with oral epithelial cells, PLoS One 6 (2011) e17046.

[10] J. Berman, P.E. Sudbery, Candida Albicans: a molecular revolution built on lessons from budding yeast, Nat. Rev. Genet. 3 (2002) 918-930.

[11] S.P. Saville, A.L. Lazzell, C. Monteagudo, J.L. Lopez-Ribot, Engineered control of cell morphology in vivo reveals distinct roles for yeast and filamentous forms of Candida albicans during infection, Eukaryot. Cell 2 (2003) 1053-1060.

[12] C.G. Pierce, J.L. Lopez-Ribot, Candidiasis drug discovery and development: new approaches targeting virulence for discovering and identifying new drugs, Expet Opin. Drug Discov. 8 (2013) 1117-1126.

[13] D. Araujo, M. Henriques, S. Silva, Portrait of Candida species biofilm regulatory network genes, Trends Microbiol. 25 (2017) 62-75.

[14] S. Mendelsohn, M. Pinsky, Z. Weissman, D. Kornitzer, Regulation of the Candida albicans hypha-inducing transcription factor Ume6 by the CDK1 cyclins Cln3 and Hgc1, mSphere (2017) 2.

[15] Z. Palková, L. Váchová, Yeast cell differentiation: lessons from pathogenic and nonpathogenic yeasts, Semin. Cell Dev. Biol. 57 (2016) 110-119.

[16] L. Scorzoni, E. de Paula, A.C. Silva, C.M. Marcos, P.A. Assato, W.C. de Melo, H.C. de Oliveira, et al., Antifungal therapy: new advances in the understanding and treatment of mycosis, Front. Microbiol. 8 (2017) 36.

[17] A.E. Clatworthy, E. Pierson, D.T. Hung, Targeting virulence: a new paradigm for antimicrobial therapy, Nat. Chem. Biol. 3 (2007) 541-548.

[18] C.G. Pierce, A.K. Chaturvedi, A.L. Lazzell, A.T. Powell, S.P. Saville, S.F. McHardy, et al., A novel small molecule inhibitor of Candida albicans biofilm formation, filamentation and virulence with low potential for the development of resistance, NPJ Biofilms Microb. (2015) 1.

[19] Y. Li, W. Chang, M. Zhang, Z. Ying, H. Lou, Natural product solasodine-3-O- $\beta-D-$ glucopyranoside inhibits the virulence factors of Candida albicans, FEMS Yeast Res. (2015) 15.

[20] C.E. Graham, M.R. Cruz, D.A. Garsin, M.C. Lorenz, Enterococcus faecalis bacteriocin EntV inhibits hyphal morphogenesis, biofilm formation, and virulence of Candida albicans, Proc. Natl. Acad. Sci. U. S. A. 114 (2017) 4507-4512.

[21] P.I. Diaz, L.D. Strausbaugh, A. Dongari-Bagtzoglou, Fungal-bacterial interactions and their relevance to oral health: linking the clinic and the bench, Front Cell Infect Microbiol. 4 (2014) 101.

[22] D.K. Morales, D.A. Hogan, Candida albicans interactions with bacteria in the context of human health and disease, PLoS Pathog. 6 (2010) e1000886.

[23] C. Heinemann, J.E. van Hylckama Vlieg, D.B. Janssen, H.J. Busscher, H.C. van der Mei, G. Reid, Purification and characterization of a surface-binding protein from Lactobacillus fermentum RC-14 that inhibits adhesion of Enterococcus faecalis 1131 FEMS Microbiol. Lett. 190 (2000) 177-180.

[24] S. Elahi, G. Pang, R. Ashman, R. Clancy, Enhanced clearance of Candida albicans from the oral cavities of mice following oral administration of Lactobacillus acidophilus, Clin. Exp. Immunol. 141 (2005) 29-36.

[25] D. Mailänder-Sánchez, C. Braunsdorf, C. Grumaz, C. Müller, S. Lorenz, P. Stevens, et al., Antifungal defense of probiotic Lactobacillus rhamnosus GG is mediated by blocking adhesion and nutrient depletion, PLoS One 12 (2017) e0184438.

[26] S. Boris, C. Barbés, Role played by lactobacilli in controlling the population of vaginal pathogens, Microb. Infect. 2 (2000) 543-546.

[27] R.D. Rossoni, B.B. Fuchs, P.P. de Barros, M.D. Velloso, A.O. Jorge, J.C. Junqueira, et al., Lactobacillus paracasei modulates the immune system of Galleria mellonella and protects against Candida albicans infection, PLoS One 12 (2017) e0173332.

[28] J.J. Coleman, I. Okoli, G.P. Tegos, E.B. Holson, F.F. Wagner, M.R. Hamblin, et al., Characterization of plant-derived saponin natural products against Candida albicans, ACS Chem. Biol. 5 (2010) 321-332.

[29] J.J. Coleman, T. Komura, J. Munro, M.P. Wu, R.R. Busanelli, A.N. Koehler, et al.,
Activity of caffeic acid phenethyl ester in Caenorhabditis elegans, Future Med. Chem. 8 (17) (2016) 2033-2046.

[30] J.J. Ewbank, O. Zugasti, C. elegans: model host and tool for antimicrobial drug discovery, Dis Model Mech 4 (2011) 300-304.

[31] J. Breger, B.B. Fuchs, G. Aperis, T.I. Moy, F.M. Ausubel, E. Mylonakis, Antifungal chemical compounds identified using a C. elegans pathogenicity assay, PLoS Pathog. 3 (2007) e18.

[32] L. Scorzoni, M.P. de Lucas, A.C. Mesa-Arango, A.M. Fusco-Almeida, E. Lozano, M. Cuenca-Estrella, et al., Antifungal efficacy during Candida krusei infection in non-conventional models correlates with the yeast in vitro susceptibility profile, PLoS One 8 (2013) e60047.

[33] R. Pukkila-Worley, A.Y. Peleg, E. Tampakakis, E. Mylonakis, Candida albicans hyphal formation and virulence assessed using a Caenorhabditis elegans infection model, Eukaryot. Cell 8 (2009) 1750-1758.

[34] E. Tampakakis, A.Y. Peleg, E. Mylonakis, Interaction of Candida albicans with an intestinal pathogen, Salmonella enterica serovar Typhimurium, Eukaryot. Cell 8 (2009) 732-737.

[35] R.D. Rossoni, P.P. de Barros, J.A. de Alvarenga, F.C. Ribeiro, M.D.S. Velloso, B.B. Fuchs, et al., Antifungal activity of clinical Lactobacillus strains against Candida albicans biofilms: identification of potential probiotic candidates to prevent oral candidiasis, Biofouling (2018) 1-14.

[36] S.F. Vilela, J.O. Barbosa, R.D. Rossoni, J.D. Santos, M.C. Prata, A.L. Anbinder, et al., Lactobacillus acidophilus ATCC 4356 inhibits biofilm formation by C. albicans and attenuates the experimental candidiasis in Galleria mellonella, Virulence 6 (2015) 29-39.

[37] F.C. Ribeiro, P.P. de Barros, R.D. Rossoni, J.C. Junqueira, A.O. Jorge, Lactobacillus rhamnosus inhibits Candida albicans virulence factors in vitro and modulates immune system in Galleria mellonella, J. Appl. Microbiol. 122 (2017) 201-211.

[38] H. Nailis, S. Kucharikova, M. Ricicova, P. Van Dijck, D. Deforce, H. Nelis, et al., Real-time PCR expression profiling of genes encoding potential virulence factors in Candida albicans biofilms: identification of model-dependent and -independent gene expression, BMC Microbiol. 10 (2010) 114.

[39] D. Hnisz, A.F. Bardet, C.J. Nobile, A. Petryshyn, W. Glaser, U. Schock, et al., A histone deacetylase adjusts transcription kinetics at coding sequences during Candida albicans morphogenesis, PLoS Genet. 8 (2012) e1003118.

[40] K.J. Livak, T.D. Schmittgen, Analysis of relative gene expression data using real time quantitative PCR and the 2(-Delta Delta C(T)) Method, Methods 25 (2001) 402-408.

[41] S. Oh, G.W. Go, E. Mylonakis, Y. Kim, The bacterial signalling molecule indole attenuates the virulence of the fungal pathogen Candida albicans, J. Appl. Microbiol. 113 (2012) 622-628.

[42] Y. Kim, E. Mylonakis, Caenorhabditis elegans immune conditioning with the probiotic bacterium Lactobacillus acidophilus strain NCFM enhances gram-positive immune responses, Infect. Immun. 80 (2012) 2500-2508.

[43] A.Y. Peleg, E. Tampakakis, B.B. Fuchs, G.M. Eliopoulos, R.C. Moellering Jr., E. Mylonakis, Prokaryote-eukaryote interactions identified by using Caenorhabditis elegans, Proc. Natl. Acad. Sci. U. S. A. 105 (2008) 14585-14590.

[44] Y. Rivera-Espinoza, Y. Gallardo-Navarro, Non-dairy probiotic products, Food Microbiol. 27 (2010) 1-11.

[45] V.H. Matsubara, Y. Wang, H.M. Bandara, M.P. Mayer, L.P. Samaranayake, Probiotic lactobacilli inhibit early stages of Candida albicans biofilm development by reducing their growth, cell adhesion, and filamentation, Appl. Microbiol. Biotechnol. 100 (2016) 6415-6426.

[46] J. Borrero, E. Kelly, P.M. O'Connor, P. Kelleher, C. Scully, P.D. Cotter, et al., Purification, characterization and heterologous production of plantaricyclin $\mathrm{A}$, a novel circular bacteriocin produced by Lactobacillus plantarum NI326, Appl. Environ. Microbiol. (2017).

[47] K. Surachat, U. Sangket, P. Deachamag, W. Chotigeat, In silico analysis of protein toxin and bacteriocins from Lactobacillus paracasei SD1 genome and available online databases, PLoS One 12 (2017) e0183548.

[48] P. Mastromarino, P. Brigidi, S. Macchia, L. Maggi, F. Pirovano, V. Trinchieri, et al., Characterization and selection of vaginal Lactobacillus strains for the preparation of vaginal tablets, J. Appl. Microbiol. 93 (2002) 884-893.

[49] J.A. Younes, H.C. van der Mei, E. van den Heuvel, H.J. Busscher, G. Reid, Adhesion forces and coaggregation between vaginal staphylococci and lactobacilli, PLoS One 7 (2012) e36917.

[50] M.S. do Carmo, F.M. Noronha, M.O. Arruda, E.P. Costa, M.R. Bomfim, A.S. Monteiro, et al., Lactobacillus fermentum ATCC 23271 displays In vitro inhibitory activities against Candida spp, Front. Microbiol. 7 (2016) 1722.

[51] L.N. Nguyen, L.C. Lopes, R.J. Cordero, J.D. Nosanchuk, Sodium butyrate inhibits pathogenic yeast growth and enhances the functions of macrophages, $\mathrm{J}$. Antimicrob. Chemother. 66 (2011) 2573-2580.

[52] M.C. Noverr, G.B. Huffnagle, Regulation of Candida albicans morphogenesis by fatty acid metabolites, Infect. Immun. 72 (2004) 6206-6210.

[53] M.C. Verdenelli, M.M. Coman, C. Cecchini, S. Silvi, C. Orpianesi, A. Cresci, Evaluation of antipathogenic activity and adherence properties of human Lactobacillus strains for vaginal formulations, J. Appl. Microbiol. 116 (2014) 1297-1307.

[54] S.D. Todorov, D.N. Furtado, S.M. Saad, Gombossy de Melo Franco BD. Bacteriocin production and resistance to drugs are advantageous features for Lactobacillus acidophilus La-14, a potential probiotic strain, N. Microbiol. 34 (2011) 357-370.

[55] B. Kaur, P.P. Balgir, B. Mittu, B. Kumar, N. Garg, Biomedical applications of fermenticin HV6b isolated from Lactobacillus fermentum HV6b MTCC10770, BioMed Res. Int. 2013 (2013) 168438.

[56] A. Schweizer, S. Rupp, B.N. Taylor, M. Rollinghoff, K. Schroppel, The TEA/ATTS transcription factor CaTec1p regulates hyphal development and virulence in 
Candida albicans, Mol. Microbiol. 38 (2000) 435-445.

[57] M.S. Skrzypek, M.B. Arnaud, M.C. Costanzo, D.O. Inglis, P. Shah, G. Binkley, et al., New tools at the Candida Genome Database: biochemical pathways and full-text literature search, Nucleic Acids Res. 38 (2010) D428-D432.

[58] M. Banerjee, D.S. Thompson, A. Lazzell, P.L. Carlisle, C. Pierce, C. Monteagudo, et al., UME6, a novel filament-specific regulator of Candida albicans hyphal extension and virulence, Mol. Biol. Cell 19 (2008) 1354-1365.

[59] P.L. Carlisle, M. Banerjee, A. Lazzell, C. Monteagudo, J.L. Lopez-Ribot, D. Kadosh, Expression levels of a filament-specific transcriptional regulator are sufficient to determine Candida albicans morphology and virulence, Proc. Natl. Acad. Sci. U. S. A. 106 (2009) 599-604.

[60] U. Zeidler, T. Lettner, C. Lassnig, M. Muller, R. Lajko, H. Hintner, et al., UME6 is a crucial downstream target of other transcriptional regulators of true hyphal development in Candida albicans, FEMS Yeast Res. 9 (2009) 126-142.

[61] R. Gordan, S. Pyne, M.L. Bulyk, Identification of cell cycle-regulated, putative hyphal genes in Candida albicans, Pac Symp Biocomput. (2012) 299-310.

[62] S.M. Noble, B.A. Gianetti, J.N. Witchley, Candida albicans cell-type switching and functional plasticity in the mammalian host, Nat. Rev. Microbiol. 15 (2017) 96-108.

[63] T. Vila, J.A. Romo, C.G. Pierce, S.F. McHardy, S.P. Saville, J.L. Lopez-Ribot, Targeting Candida albicans filamentation for antifungal drug development,
Virulence 8 (2017) 150-158.

[64] X. Tan, B.B. Fuchs, Y. Wang, W. Chen, G.J. Yuen, R.B. Chen, et al., The role of Candida albicans SPT20 in filamentation, biofilm formation and pathogenesis, PLoS One 9 (2014) e94468.

[65] I.Y. Hwang, E. Koh, A. Wong, J.C. March, W.E. Bentley, Y.S. Lee, et al., Engineered probiotic Escherichia coli can eliminate and prevent Pseudomonas aeruginosa gut infection in animal models, Nat. Commun. 8 (2017) 15028.

[66] M. Li, K. Lee, M. Hsu, G. Nau, E. Mylonakis, B. Ramratnam, Lactobacillus-derived extracellular vesicles enhance host immune responses against vancomycin-resistant enterococci, BMC Microbiol. 17 (2017) 66.

[67] K. Neuhaus, M.C. Lamparter, B. Zolch, R. Landstorfer, S. Simon, B. Spanier, et al., Probiotic Enterococcus faecalis Symbioflor(R) down regulates virulence genes of EHEC in vitro and decrease pathogenicity in a Caenorhabditis elegans model, Arch. Microbiol. 199 (2017) 203-213.

[68] M. Zhou, H. Yu, X. Yin, P.M. Sabour, W. Chen, J. Gong, Lactobacillus zeae protects Caenorhabditis elegans from enterotoxigenic Escherichia coli-caused death by in hibiting enterotoxin gene expression of the pathogen, PLoS One 9 (2014) e89004.

[69] N. Pujol, O. Zugasti, D. Wong, C. Couillault, C.L. Kurz, H. Schulenburg, et al., Antifungal innate immunity in C. elegans is enhanced by evolutionary diversification of antimicrobial peptides, PLoS Pathog. 4 (2008) e1000105. 